\title{
Revision of Bubble Bursting: Universal Scaling Laws of Top Jet Drop Size and Speed
}

\author{
Alfonso M. Gañán-Calvo* \\ Departamento de Ingeniería Aerospacial y Mecánica de Fluidos, Universidad de Sevilla, \\ Camino de los Descubrimientos s/n 41092, Spain
}

(Received 12 July 2017; revised manuscript received 17 August 2017; published 16 November 2017)

\begin{abstract}
The collapse of a bubble of radius $R_{o}$ at the surface of a liquid generating a liquid jet and a subsequent first drop of radius $R$ is universally scaled using the Ohnesorge number $\mathrm{Oh}=\mu /\left(\rho \sigma R_{o}\right)^{1 / 2}$ and a critical value $\mathrm{Oh}^{*}$ below which no droplet is ejected; $\rho, \sigma$, and $\mu$ are the liquid density, surface tension, and viscosity, respectively. First, a flow field analysis at ejection yields the scaling of $R$ with the jet velocity $V$ as $R / l_{\mu} \sim\left(V / V_{\mu}\right)^{-5 / 3}$, where $l_{\mu}=\mu^{2} /(\rho \sigma)$ and $V_{\mu}=\sigma / \mu$. This resolves the scaling problem of curvature reversal, a prelude to jet formation. In addition, the energy necessary for the ejection of a jet with a volume and averaged velocity proportional to $R_{o} R^{2}$ and $V$, respectively, comes from the energy excess from the total available surface energy, proportional to $\sigma R_{o}^{2}$, minus the one dissipated by viscosity, proportional to $\mu\left(\sigma R_{o}^{3} / \rho\right)^{1 / 2}$. Using the scaling variable $\varphi=\left(\mathrm{Oh}^{*}-\mathrm{Oh}\right) \mathrm{Oh}^{-2}$, it yields $V / V_{\mu}=k_{v} \varphi^{-3 / 4}$ and $R / l_{\mu}=k_{d} \varphi^{5 / 4}$, which collapse published data since 1954 and resolve the scaling of $R$ and $V$ with $k_{v}=16, k_{d}=0.6$, and $\mathrm{Oh}^{*}=0.043$ when gravity effects are negligible.
\end{abstract}

DOI: 10.1103/PhysRevLett.119.204502

Natural aerosols present in the atmosphere and cloud condensation nuclei $(\mathrm{CCN})$ arise in a significant proportion from the breakup of bubbles bursting at the sea surface, producing a plethora of droplet sizes from the nanometric to the hundred-micrometer size range [1], depending on the breakup mechanism and parent bubble size. The importance of $\mathrm{CCN}$ in climate (Earth's radiation balance and precipitation) is paramount. Thus, changing properties of surface seawater owing to climate change and human actions impact on aerosol generation at the sea surface; this is a key phenomenon that should be completely elucidated. Other substantial sources of complex aerosols occur when an organic liquid is heated (oil dip frying is a worldwide basic food processing for humans), by burning biomass, or by smoking: In these processes, cellulose and more complex biochemicals melt into very low surface tension liquids, releasing vapors from their bulk in the form of microbubbles that eventually burst and generate aerosols [2]. In most cases, the visible smoke from fires and their toxicity come from these aerosols and their burning. In addition, part of the ashes from volcanoes has the same origin. Besides, gas injection in bioreactors, which compulsorily involves bubbling, produces harmful effects on cultivated cells when bubbles reach the surface due to the mechanical action of bubble bursting [3,4]. In a more relaxing context, the organoleptic properties of sparkling wines and the sensorial experiences associated to their consumption depend to a great extent on the aroma carried by droplets ejected from the bursting bubbles $[5,6]$. Thus, this is one of the most fundamental fluidic phenomena at the microscopic scale, and its overwhelming transversal impact across disciplines has fueled an active research once its practical importance was perceived beyond initial curiosity.
The objectives of the analysis of this phenomenon are, fundamentally, twofold: (i) to determine the size of the emitted droplet and its velocity for a given set of liquid properties (density $\rho$, viscosity $\mu$, and surface tension $\sigma$ ) and parent bubble size and (ii) to pinpoint the critical size of the latter below which no droplet is ejected. When the parent bubble size is sufficiently large, or the viscosity sufficiently low for an energetic wave to collapse at the axis, a couple of Worthington jets [7] are formed, i.e., one ejecting a capillary jet out of the cavity, and the other injecting momentum into the liquid below the cavity, sometimes engulfing a small bubble [8]. Some recent works $[5,6,10,11]$ have unveiled the subtle balance between the advance of the capillary wave front coming from the expansion of the initial rim formed after the film cap bursting and its viscous damping when the wave collapses at the axis (Fig. 1). The front progression,

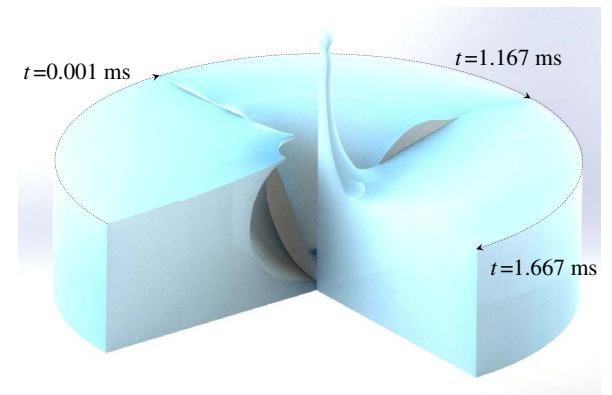

FIG. 1. A 3D rendering and composition of three fundamental instants of a bubble collapse taken from Fig. 1 of MacIntyre [13]: $t \sim 0$ (just after film rupture), 1.17 (onset of jet ejection), and $1.67 \mathrm{~ms}$ (just before the first droplet detachment). (Courtesy of B. Gañán-Riesco, Ingeniatrics Tec. S.L.) 
common to other related phenomena [12], has been observed since early studies [13]. The overall speed of the capillary wave front should be $v_{c} \sim(\sigma k / \rho)^{1 / 2}$, while the dominant wave number $k$ should be proportional to $R_{o}^{-1}$. Besides, the viscous damping rate of the capillary wave, $t_{D}^{-1} \sim k^{2} \mu / \rho$, should be smaller than the progression rate of the wave, i.e., $\left(\sigma / \rho R_{o}^{3}\right)^{1 / 2}>\mu /\left(\rho R_{o}^{2}\right)$ (discarding constants). Thus, one has that the Ohnesorge number $\mathrm{Oh}=$ $\mu /\left(\rho \sigma R_{o}\right)^{1 / 2}$ should be smaller than a critical one $\mathrm{Oh}^{*}$ for jetting to occur. Walls, Henaux, and Bird [11] experimentally calculated that critical $\mathrm{Oh}^{*}$ as a function of the Bond number $\mathrm{Bo}=\rho g R_{o}^{2} / \sigma$.

Ghabache et al. $[5,6]$ suggested an interesting explanation on the kernel of the ejection phenomenon. They determined the length scale as a function of the time to collapse, found a geometrical self-similarity of the collapsing phenomenon for a liquid viscosity above a certain range, and proposed a general scaling law of the ejected droplet size as a function of the ejection velocity, liquid properties, and gravity [6]. Their measurements deviated significantly from their model for higher viscosities or smaller bubble sizes tested, but they provide the best published source of experimental data on the phenomenon so far.

Here, a definitive and accurate solution of the top droplet size and its ejection velocity as a function of the bubble radius and Newtonian liquid properties, valid for any value of $\mathrm{Oh}<\mathrm{Oh}^{*}$, is proposed. The focusing role of viscosity is finally underpinned and quantified.

First, consider the widely recognized and numerically described concentration of viscous stresses taking place at the vicinity of the axis close to the surface when the capillary wave front collapses. Numerical simulations $[2,4,14]$ show a rather complex yet totally repeatable flow structure initially comprising two evolving vortices near the axis: One is projected backwards from the surface, and the other pushes the surface outwards in the form of a capillary jet [14]. When the capillary wave front collapses onto the axis, the interaction of the vortical structure with the interface and the complexity of the nonlinear capillary wave front can give rise to complex geometries in the local liquid surface, leading in many cases to either single or multiple gas engulfment in the form of tiny bubbles in addition to the liquid ejection. However, when the ejection initiates, a basic overarching picture emerges for the dynamically balanced flow structure. It can be reduced to relatively simple terms; one important realization here is that the maximum velocity takes place at the very moment where the first dimple forms at the bottom of the collapsing cavity [15] and that this velocity should be sufficient to finally eject and detach a top droplet. Figure 2 illustrates this instant with its characteristic radial and axial length and velocity scales $R$ and $L$ and $V^{\prime}$ and $V$, respectively ( $R$ is the radius of the first emitted droplet, and $V$ is the initial velocity of the jet).

Neglecting the effects of gravity for the bubble sizes of interest here $($ Bo $\ll 1)$, one can analyze the momentum

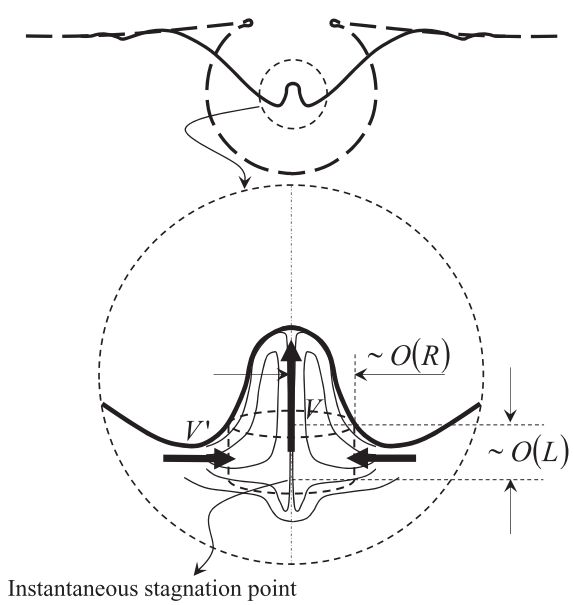

FIG. 2. Onset of ejection. The complex vortical structure was already described [14]. $V$ and $V^{\prime}$ stand for the scales of the jet speed and the radial surface velocity, respectively. $R$ is the radial scale (final droplet radius). Thin continuous lines represent vorticity isolines. A cylindrical surface with characteristic axial and radial scales $L$ and $R$, respectively, is indicated with dashed lines.

equation for the liquid particles at the liquid surface close to the axis at the onset of jet ejection [16]:

$$
\rho\left(\mathbf{v}_{t}+\mathbf{v} \cdot \boldsymbol{\nabla} \mathbf{v}\right)+\sigma \boldsymbol{\nabla}\left(\boldsymbol{\nabla}_{s} \cdot \mathbf{n}\right)-\mu \boldsymbol{\nabla}^{2} \mathbf{v} \simeq \mathbf{0},
$$

where $\mathbf{v}$ and $\mathbf{n}$ are the liquid velocity and the normal unit vector at the surface, respectively. Subscripts $t$ and $s$ indicate the partial derivative with time and the surface derivative, respectively. The flow configuration (Fig. 2) leads to the following scales: (i) local and convective acceleration forces (both are comparable in this phenomenon), $\rho \mathbf{v}_{t} \sim \rho \mathbf{v} \cdot \nabla \mathbf{v} \sim O\left(\rho V^{2} / L\right)$; (ii) surface tension force, $\sigma \nabla\left(\nabla_{s} \cdot \mathbf{n}\right) \sim O\left(\sigma R^{-2}\right)$; and (iii) viscous force, $\mu \nabla^{2} \mathbf{v} \sim O\left(\mu V^{\prime} L^{-2}\right)$. On the other hand, considering the fixed cylindrical control volume indicated by dashed lines in Fig. 2, integral liquid mass continuity demands $O\left(V R^{2}\right) \sim O\left(V^{\prime} L R\right)$. Since all terms of the momentum equation become of the same order at the onset of ejection, a fact providing two additional conditions, one obtains the following three relationships with the initial jet velocity $V$ :

$$
\begin{aligned}
& R / l_{\mu} \sim\left(V / V_{\mu}\right)^{-5 / 3}, \\
& L / l_{\mu} \sim\left(V / V_{\mu}\right)^{-4 / 3}, \\
& V^{\prime} / V_{\mu} \sim\left(V / V_{\mu}\right)^{2 / 3},
\end{aligned}
$$

where $V_{\mu}=\sigma \mu^{-1}$. These are universal relationships that should hold for a variety of phenomena involving capillary surface collapse and jet ejection [12,17], a study that is given elsewhere [18]. Focusing on bubble bursting, a number of publications disclose both the jet speed close to the incept of ejection and the final top droplet size, providing all liquid properties $[6,13,14,19,20]$. The data 


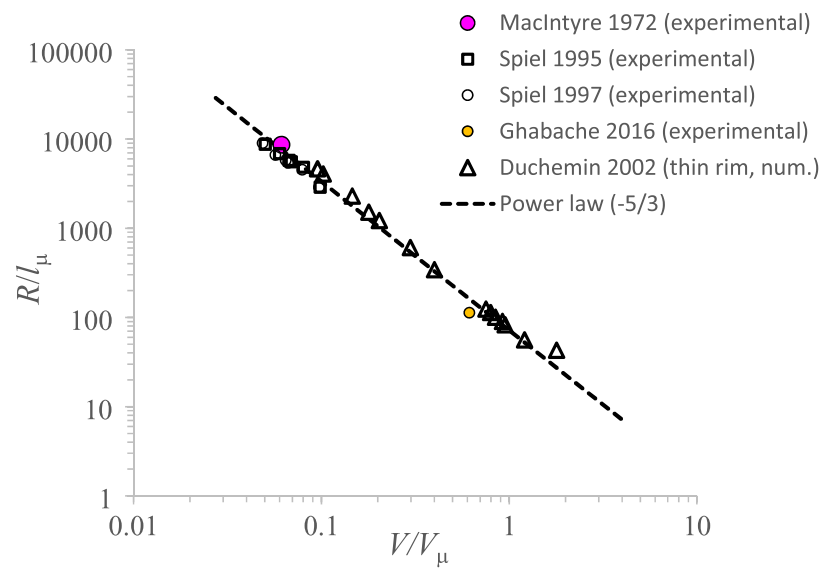

FIG. 3. Nondimensional droplet radius $R / l_{\mu}$ as a function of the nondimensional axial jet velocity at ejection $V / V_{\mu}$ reported in the literature $[6,13,14,19,20]$ for $\mathrm{Bo}<0.1$. The more realistic thin rim simulations of Duchemin et al. [14] are used.

collected for Bo $<0.1$ are plotted in Fig. 3, showing an excellent agreement with the proposed scaling law.

The initial jet speed $V$ provides a measure of the surplus of mechanical energy $\Delta E$ forming the jet after the collapse singularity. That surplus comes from the available energy after bubble bursting, i.e., the surface energy proportional to $\sigma R_{o}^{2}$, minus the total viscous dissipation associated to the motion induced by the capillary wave after bursting, proportional to $\left(\mu v_{c}^{2} / R_{o}^{2}\right) R_{o}^{3} t_{c} \sim \mu\left(\sigma R_{o}^{3} / \rho\right)^{1 / 2}$; here, the total time of the process is assumed proportional to the capillary time $t_{c} \sim\left(R_{o} / v_{c}\right)=\left(\rho R_{o}^{3} / \sigma\right)^{1 / 2}$. Using a single physically meaningful proportionality constant in the balance (here, $\left.\mathrm{Oh}^{*}\right)$ and neglecting other overall prefactors, one can write

$\Delta E \sim\left[\mathrm{Oh}^{*} \sigma R_{o}^{2}-\mu\left(\sigma R_{o}^{3} / \rho\right)^{1 / 2}\right]=\sigma R_{o}^{2}\left(\mathrm{Oh}^{*}-\mathrm{Oh}\right)$,

where $\mathrm{Oh}^{*}$ provides the right constant to fulfil $\Delta E=0$ when the critical condition for droplet ejection is met. Next, this energy excess is invested in the generation of a jet with sufficient kinetic energy to overcome the recoil effect of the final stages of bursting (see, e.g., $[6,13,14,21]$ ). A fundamental observation here is that this recoil takes place when the uprising liquid column reaches a length proportional to $R_{o}$ (see the excellent illustration on this fact provided by Fig. 1 in Ghabache and Séon [6]): The top droplet must detach before the liquid front reaches this length. Thus, the total amount of kinetic energy required for drop ejection should be proportional to $\rho V^{2}$ times the total jet volume, proportional to $R^{2} R_{o}$. Using scaling (2) for $R$, that yields

$\rho V^{2} R^{2} R_{o} \sim \Delta E \Rightarrow\left(V / V_{\mu}\right)^{-4 / 3} \sim \frac{\sigma R_{o} \rho}{\mu^{2}}\left(\mathrm{Oh}^{*}-\mathrm{Oh}\right)$,

that can be reduced to

$$
\frac{V}{V_{\mu}} \sim \varphi^{-3 / 4}
$$

defining $\varphi=\left[\left(\mathrm{Oh}^{*} / \mathrm{Oh}\right)-1\right] \mathrm{Oh}^{-1}$.
TABLE I. Liquid properties from published experiments. SW, seawater. $\mathrm{W}+\mathrm{G}$, water-glycerol mixtures. Properties of mixtures used by Ghabache et al. [27] can be directly seen in their Table I.

\begin{tabular}{lcccc}
\hline \hline Liquid & Ref. & \multicolumn{1}{c}{$\rho\left(\mathrm{kg} \mathrm{m}^{-3}\right) \sigma\left(\mathrm{N} \mathrm{m}^{-1}\right) \mu(\mathrm{Pa} \mathrm{s})$} \\
\hline $\mathrm{SW} 4{ }^{\circ} \mathrm{C}$ & {$[23]$} & 1028 & 0.0755 & 0.00167 \\
$\mathrm{SW} 16^{\circ} \mathrm{C}$ & {$[24]$} & 1025 & 0.0736 & 0.00112 \\
$\mathrm{SW} \mathrm{20}{ }^{\circ} \mathrm{C}$ & {$[23,25]$} & 1025 & 0.0734 & 0.00108 \\
$\mathrm{SW} \mathrm{30}{ }^{\circ} \mathrm{C}$ & {$[19,24]$} & 1024 & 0.071 & 0.00098 \\
Water & {$[6,13,20,26]$} & 1000 & 0.072 & 0.001 \\
$\mathrm{~W}+30 \% \mathrm{G} \mathrm{25} 5^{\circ} \mathrm{C}$ & {$[6]$} & 1078 & 0.067 & 0.0021 \\
$\mathrm{~W}+50 \% \mathrm{G} \mathrm{25}{ }^{\circ} \mathrm{C}$ & {$[6]$} & 1130 & 0.065 & 0.0044 \\
$\mathrm{~W}+60 \% \mathrm{G} \mathrm{30} 0^{\circ} \mathrm{C}$ & {$[6]$} & 1156 & 0.064 & 0.0062 \\
$\mathrm{~W}+60 \% \mathrm{G} \mathrm{25}{ }^{\circ} \mathrm{C}$ & {$[6]$} & 1156 & 0.064 & 0.0074 \\
$\mathrm{~W}+60 \% \mathrm{G} \mathrm{20}{ }^{\circ} \mathrm{C}$ & {$[6]$} & 1156 & 0.064 & 0.0097 \\
\hline \hline
\end{tabular}

Two hundred measurements have been directly taken [22] from the available literature on the phenomenon since 1954 that disclose both bubble and droplet size values $[6,11,13,14,19,20,23-27]$. With the exception of data from Duchemin et al. [14], all data are experimental, and the properties of liquids used are given in Table I. First, we compare the proposed scaling of $V$ with the axial jet velocities taken from experiments $[5,6,19,20]$ and numerical simulations by Duchemin et al. (see Fig. 12 in Ref. [14]) at the axial point where these authors measure the jet velocity (when the jet tip reaches the mean water level). Figure 4 shows the collapse of $V / V_{\mu}$ values for Bo $<0.1$ around the model. The fitting yields $V / V_{\mu}=k_{v} \varphi^{-3 / 4}$ with $k_{v} \simeq 16$ and $\mathrm{Oh}^{*}=0.043$ (the latter about $14 \%$ larger than the value found by Walls, Henaux, and Bird [11]). The excellent agreement grants a full support to the proposed model. Even stronger evidence is afforded by Fig. 5, where the nondimensional droplet radius $R / l_{\mu}$ is plotted as a function of the scaling variable $\varphi$. The master curve

$$
R / l_{\mu}=k_{d} \varphi^{5 / 4}
$$

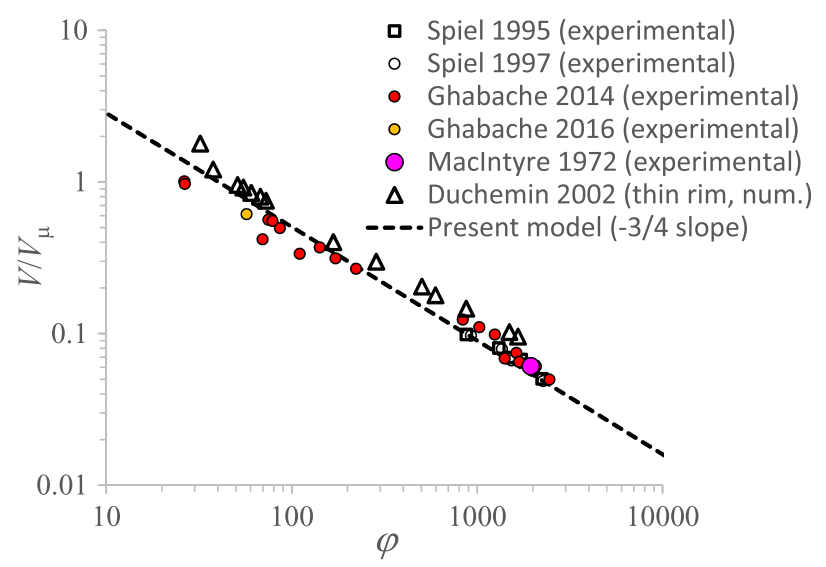

FIG. 4. Axial jet velocities reported in the literature $[5,6,14,19,20]$ for Bo $<0.1$. 


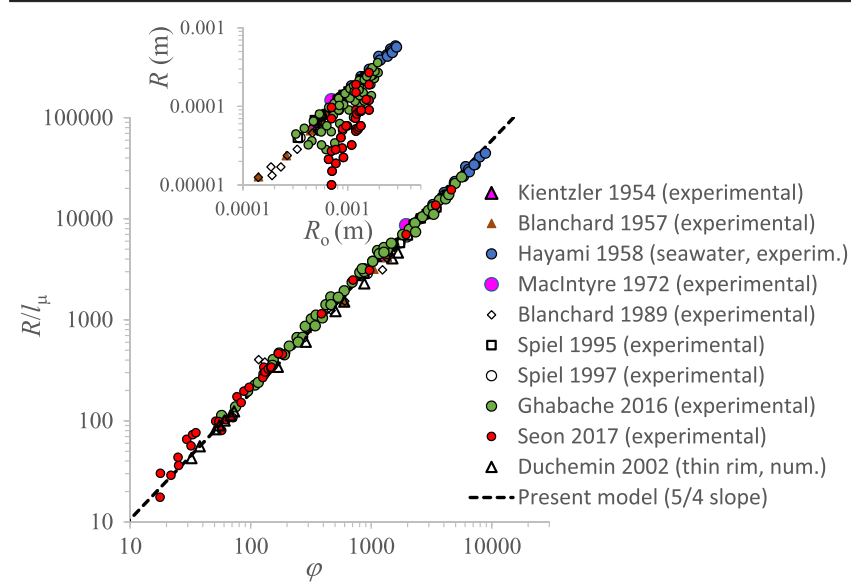

FIG. 5. Universal scaling of experimental results according to the proposed model. The data are taken from figures in Refs. [6,11,13,14,19,20,23-27]; in particular, all data from Séon and Liger-Belair [see [21], Figs. 16 and 19(b) and the higher viscosity cases of Fig. 17(a)] have been used. The standard deviation of all data from the model (excluding wide rim from Ref. [14]) is $13.6 \%$. The inset shows the raw data. Note that this figure is not just the composition of Figs. 3 and 4: It shows many more points, since many experimental data sets reported in the literature disclose the ejected droplet size but not the speed of the jet's tip.

as required by the scaling law (2) is also shown. The extraordinary collapse of all data around the proposed model for $k_{d}=0.6$, with a standard deviation below $15 \%$, was a surprise for the author himself. Finally, the characteristic axial length $L$ can be defined without limitation as $L=\varphi l_{\mu}=\left(\mathrm{Oh}^{*}-\mathrm{Oh}\right) R_{o} \quad$ in consistency with scalings (2) and (3) and the values of $k_{v}$ and $k_{d}$. Naturally, for $\mathrm{Oh} \ll \mathrm{Oh}^{*}$, the universal scaling laws reduce to $V=16 \mathrm{Oh}^{*-3 / 4} \mathrm{Oh}^{3 / 2} V_{\mu}$ and $R=0.6 \mathrm{Oh}^{* 5 / 4} \mathrm{Oh}^{-5 / 2} l_{\mu}$. Interestingly, in this limit one obtains $L=\mathrm{Oh}^{*} R_{o}$, which consistently indicates that when $\mathrm{Oh} \ll \mathrm{Oh}^{*}$ the amplitude of the capillary wave collapsing onto the axis should be asymptotically proportional to $R_{o}$ with a small prefactor, as expected from the physics and the geometry of the phenomenon (see numerical simulations, e.g., $[2,14,15]$ ).

The potential importance and the impact of these findings in areas like geophysics, chemical engineering, the food industry, or any form of massive liquid handling can be easily understood. For example, the direct relationship between the distributions of marine aerosols and of bubble sizes that end up bursting at the sea surface can now be better determined. And, more importantly, the effects of human action on critical sea regions (i.e., coastal zones) for the generation of $\mathrm{CCN}$ and inland precipitation can be rightfully attributed to induced changes in seawater properties and temperature, by the direct monitoring of the temperature and surface concentration of chemical species with surfactant effects. CCN, cloud statistics, and all their fundamental impact on radiation balance and precipitation come in great extent from the structure of the smaller size tail of marine aerosol distributions [1].

This work was supported by the Ministerio de Economía, Industria y Competitividad, Plan Estatal 2013-2016 Retos, Project No. DPI2016-78887-C3-1-R. The author is indebted to Dr. Pascual Riesco-Chueca for his suggestions.

*amgc@us.es

[1] F. Veron, Annu. Rev. Fluid Mech. 47, 507 (2015).

[2] A. Teixeira, K. G. Mooney, J. S. Kruger, C. L. Williams, W. J. Suszynski, L. D. Schmidt, D. P. Schmidtb, and P. J. Dauenhauer, Energy Environ. Sci. 4, 4306 (2011).

[3] A. Handa, A. Emery, and R. E. Spier, Dev. Biol. Standardization 66, 241 (1987), edited by the International Association of Biological Standardization.

[4] J. Boulton-Stone and J. R. Blake, J. Fluid Mech. 254, 437 (1993).

[5] E. Ghabache, A. Antkowiak, C. Josserand, and T. Séon, Phys. Fluids 26, 121701 (2014).

[6] E. Ghabache and T. Séon, Phys. Rev. Fluids 1, 051901 (2016).

[7] A. M. Worthington and R. S. Cole, Phil. Trans. R. Soc. A 189, 137 (1897).

[8] Additionally, the expanding rim which initiates its evolution from an extremely thin wedge produces another bell-shaped jet around the cavity [9] which rapidly dissipates.

[9] T. T. Tran, E. G. Lee, I. S. Lee, N. S. Woo, S. M. Han, Y. J. Kim, and W. R. Hwang, Korea-Aust. Rheol. J. 28, 315 (2016).

[10] J. S. Lee, B. M. Weon, S. J. Park, J. H. Je, K. Fezzaa, and W.-K. Lee, Nat. Commun. 2, 367 (2011).

[11] P. L. L. Walls, L. Henaux, and J. C. Bird, Phys. Rev. E 92, 021002(R) (2015).

[12] A. Yarin, Annu. Rev. Fluid Mech. 38, 159 (2006).

[13] F. MacIntyre, J. Geophys. Res. 77, 5211 (1972).

[14] L. Duchemin, S. Popinet, C. Josserand, and S. Zaleski, Phys. Fluids 14, 3000 (2002).

[15] S.-C. Georgescu, J.-L. Achard, and E. Canot, Eur. J. Mech. B 21, 265 (2002).

[16] The induced gas speeds, of the order of the liquid speeds, produce negligible effects as long as $\rho_{g} \ll \rho_{l}$. Besides, the effects of gravity are negligible for the small bubble radii of interest here: The Morton number $\mathrm{Mo}=g \mu^{4} /\left(\rho \sigma^{3}\right)$ is extremely small in this problem, of the order of $10^{-11}$ to $10^{-7}[5]$.

[17] B. W. Zeff, B. Kleber, J. Fineberg, and D. P. Lathrop, Nature (London) 403, 401 (2000).

[18] A. M. Gañán-Calvo, A. S. Mohamed, J. R. Castrejón-Pita, M. A. Herrada, and A. A. Castrejón-Pita, J. Fluid Mech. (to be published).

[19] D. E. Spiel, J. Geophys. Res. 102, 5815 (1997).

[20] D. E. Spiel, J. Geophys. Res. 100, 4995 (1995).

[21] T. Séon and G. Liger-Belair, Eur. Phys. J. Spec. Top. 226, 117 (2017).

[22] The published figures used as data sources have been enlarged, and the errors associated to direct measurement 
are smaller than $2 \%$. In many cases, the authors provide their own measurement errors around the (average) values disclosed. In other cases, direct measurements from highspeed photographs given by the authors are employed. In these cases, gray level fitting and subpixel edge detection is used.

[23] D. C. Blanchard, J. Geophys. Res. 94, 10999 (1989).
[24] S. Hayami and Y. Toba, J. Oceanogr. Soc. Jpn. 14, 145 (1958).

[25] D. C. Blanchard and A. H. Woodcock, Tellus 9, 145 (1957).

[26] C. F. Kientzler, A. B. Arons, D. C. Blanchard, and A. H. Woodcock, Tellus 6, 1 (1954).

[27] E. Ghabache, G. Liger-Belair, A. Antkowiak, and T. Séon, Sci. Rep. 6, 25148 (2016). 